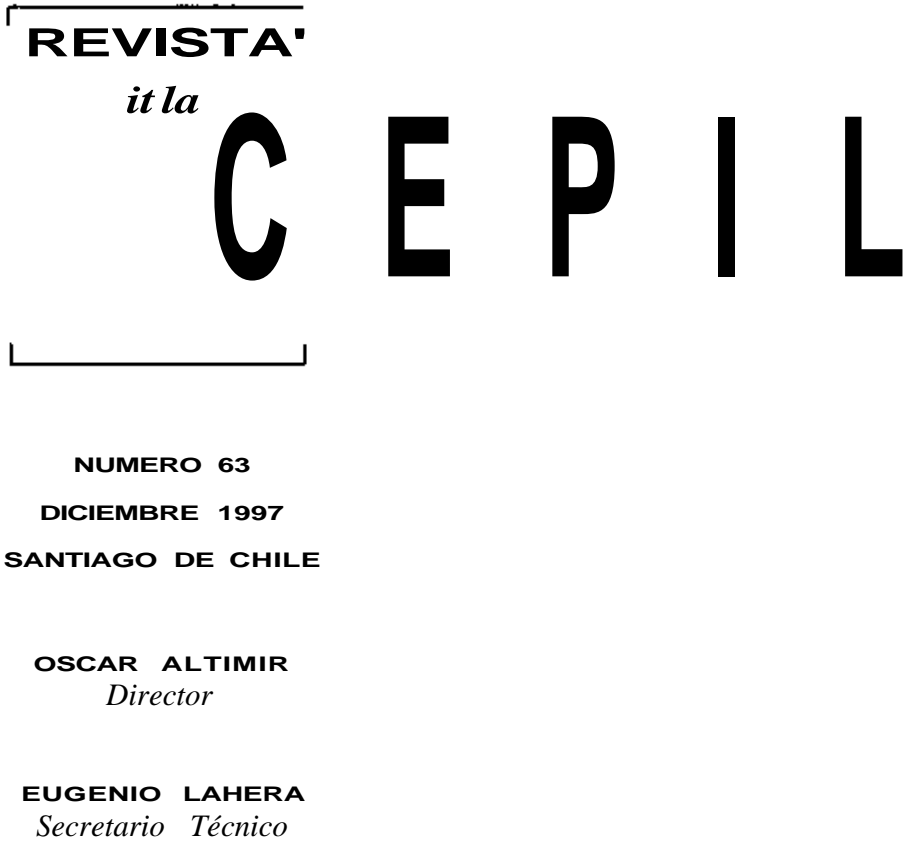

Secretario Técnico

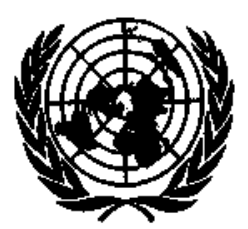

NACIONES UNIDAS 
El papel del sector público en el desarrollo latinoamericano

Ricardo Carciofi

La equidad en el presupuesto público

Juan Martin

Reformas a los sistemas de pensiones, mercado de capitales y ahorro

Andras Uthoff

Institucionalidad pública y políticas ambientales explícitas

e implícitas

Nicolo Gligo

La valoración de recursos naturales y ambientales no basada en el mercado en Centroamérica y el Caribe

Steve Shultz

Un modelo macroeconómico integrado para el Caribe

Lucio Vinhas de Souza

Virajes y derrapajes de la economía venezolana

José Miguel Benavente

¿Cuán no tradicionales son las exportaciones no tradicionales?

La experiencia de siete países de la Cuenca del Caribe

Alberto Gabriele

Apertura comercial y cambio estructural en la industria automotriz brasileña

Ruy de Quadros Carvalho, Sergio Robles Reis de Queiroz,

Flávia Luciane Consoni, loriara Costa y Janaína Pamplona da Costa

Historia evolutiva de una planta metalmecánica chilena

Jorge Katz y Héctor Vera

La importancia de la producción local y la pequeña empresa para el desarrollo de América Latina

Francisco Alburquerque

Publicaciones recientes de la CEPAL 


\section{Apertura comercial y cambio estructural en la industria automotriz brasileña}

\section{Ruy de Quadros Carvalho* \\ Sergio Robles Reis \\ de Queiroz* \\ Flávia Luciane Consoni** \\ Ionara Costa** \\ Janaína Pamplona \\ da Costa**}

* Profesor del Departamento de Política Científica y

Tecnológica del IG/UNICAMP

e investigador del Grupo de

Estudios de Empresas e

Innovación.

** Alumnos del Departamento

de Política Científica y

Tecnológica del IG/UNICAMP

e investigadora del Grupo de

Estudios de Empresas e

Innovación.
En el presente artículo se pretende sistematizar y analizar las pruebas de cambio estructural en la industria automotriz brasileña entre 1990 y 1996, procurando relacionarlas con las medidas de política económica de mayor impacto sobre el sector. Se examina el aumento explosivo de la demanda interna de vehículos automotores, sus factores determinantes e implicaciones principales, sobre todo el alcance de escalas eficientes de producción y el inicio de una ola de inversiones que se ha intensificado en los últimos tres años. Se estudia el aumento del dinamismo tecnológico del sector, consecuencia en parte de las nuevas inversiones. Se analiza un conjunto de cambios que está relacionado con la nueva inserción internacional de la industria automovilística brasileña, ya que a partir de la apertura comercial se ampliaron significativamente los coeficientes de importación de piezas y componentes o de vehículos terminados. Y por último, se analizan también algunos aspectos del llamado Régimen Automotor, cuya adopción repercutió sobre la inserción externa del sector automotor no sólo en el corto plazo, estableciendo barreras a la importación, sino también en el largo plazo, al dar a entender a las grandes empresas del sector la necesidad de invertir en la ampliación de la producción local y la mantención de niveles superiores de competitividad. 


\section{Introducción}

La industria automovilística, que comprende los segmentos del montaje de automóviles y de producción de piezas y partes, figura entre las que presentan con mayor fuerza los rasgos de la profunda reestructuración vinculada con el cambio del régimen de política industrial y de comercio exterior de Brasil. A partir de la apertura comercial, se acumulan pruebas de que este sector brasileño está modificando su inserción en el comercio internacional, acrecentando su ya elevado nivel previo de internacionalización y realizando cambios de envergadura en la base productiva, que se han reflejado en una mayor productividad. Además, éste es el único sector industrial que en la fase posterior a la apertura fue objeto de un amplio conjunto de políticas sectoriales que culminaron en la consolidación de lo que se convino en denominar Régimen Automotor.

Éste representa la primera experiencia de política sectorial de hecho, con instrumentos poderosos, orientada a administrar los "dolores" de la apertura. Motivado por estas características peculiares de la industria automovilística, este artículo persigue sistematizar y analizar las pruebas de su cambio estructural entre 1990 y 1996, procurando relacionarlas con las medidas de política económica de mayor impacto sobre el sector.

Intentamos organizar nuestra argumentación en torno a cuatro aspectos, que corresponden a las secciones del presente estudio. Ante todo se examina el aumento explosivo de la demanda interna de vehículos automotores y los factores que lo determinaron (seción
II). La nueva dimensión del mercado interno, especialmente desde el punto de vista regional, estuvo vinculada a su especialización en vehículos de tamaño pequeño y representó un cambio cualitativo en este mercado, a la vez que allanó el camino para alcanzar escalas eficientes de producción en todas las ensambladuras, por primera vez en la historia del sector automovilístico en Brasil. A nuestro juicio, el nuevo tamaño de mercado fue el factor principal de la ola de inversiones que se intensificó en 1994-1996 (sección III), aunque hay que reconocer que la apertura comercial y el Régimen Automotor operaron como catalizadores de la inversión. Las nuevas inversiones tuvieron otros dos efectos importantes en la economía de la industria automovilística brasileña: la modificación de la estructura del mercado con la entrada de otros siete competidores nuevos, y la reducción de la brecha tecnológica con las plantas situadas en los países más industrializados, que siempre marcó a las filiales brasileñas. En gran medida, todos estos fenómenos guardan relación con lo que se comienza a configurar como la nueva inserción internacional de la industria automovilística brasileña (sección IV). El proceso ha sido doloroso, con múltiples vaivenes en la conducción de la política comercial y condicionado por las estrategias de globalización de las ensambladuras. El Régimen Automotor, por su parte, ha sido decisivo para señalar a estas grandes transnacionales el equilibrio aceptable (si no deseable) entre la producción local y la importación de vehículos y piezas.

\section{II}

\section{Crecimiento y especialización del mercado interno de automóviles}

El aumento repentino del volumen del mercado y la tendencia a la especialización en vehículos pequeños

Este artículo se basa en el estudio preparado por el Grupo de Estudios de Empresas e Innovación, del Departamento de Política Científica y Tecnológica del IG/UNICAMP, para el Insituto de Investigación Económica Aplicada (IPEA), en el ámbito del proyecto "Indicadores de Productividad de la Industria Brasileña: Límites y Posibilidades". Los autores agradecen el apoyo de las siguientes sustentaron el repunte de la inversión, la modernización de las empresas y el alcance de escalas económi-

instituciones: IPEA, Secretaría de Comercio Exterior del Ministerio de Industria, Comercio y Turismo, y Asociación Nacional de Fabricantes de Vehículos Automotores (ANFAVEA) en particular en la persona del señor Sergio Sawada. El presente texto es de nuestra exclusiva responsabilidad. 
GRÁFICO 1

Brasil: Ventas internas de vehículos automotores (producción nacional), según el tipo de vehículo, 1970-1996

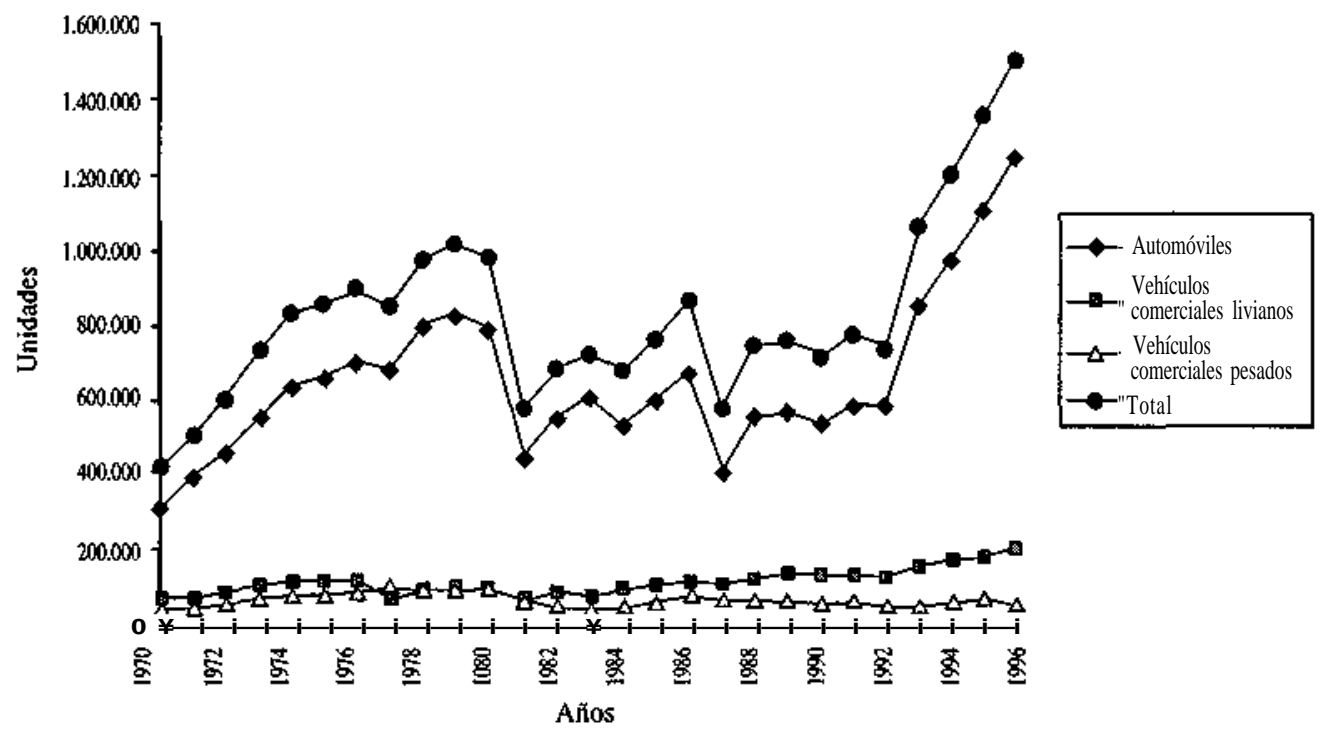

Fuente: Asociación Nacional de Fabricantes de Vehículos Automotores (ANFAVEA).

cas de producción. La integración regional propiciada por el Mercosur contribuyó a reforzar estas características. Consideramos que se trata de uno de los cambios principales ocurridos en el sector automotor en los años noventa, toda vez que la reducida dimensión de la demanda interna fue durante muchos años uno de los principales obstáculos al crecimiento y la inversión.

Tras doce años consecutivos de comportamiento inestable, en torno a un nivel de 700000 unidades anuales, el mercado brasileño de vehículos volvió a crecer en 1993 (gráfico 1). La tasa media anual de 20\% de crecimiento de las ventas internas de vehículos automotores (en unidades), en el período 1993-1996, sólo es comparable con la fase más dinámica de expansión de la demanda de comienzos de la década de 1970. Las ventas de 1.5 millones de unidades en 1996 marcaron un nuevo récord en la comercialización de vehículos producidos en Brasil. Ese año las ventas totales sumadas a las importaciones sobrepasaron la cifra de 1.7 millones de unidades. No obstante, hay que destacar que el segmento de los vehículos comerciales pesados (camiones y omnibuses) presentó una tendencia divergente de los segmentos de automóviles y vehículos comerciales, livianos, pues se ha mantenido incluso en años recientes con la demanda estancada oscilando en torno a las 60000 unidades anuales.
Debe ampliarse la definición apropiada del mercado interno para contemplar la constitución del Mercosur como mercado regional unificado. En este sentido, el ejercicio de sumar las licencias de vehículos nuevos en Brasil y Argentina revela que en realidad el mercado "interno" sobrepasó ya los dos millones de unidades en 1995, después de iniciar la década con menos de un millón de unidades (cuadro 1). Hay un cambio cualitativo derivado de este crecimiento reciente. El mercado de vehículos automotores, considerando el Mercosur, alcanzó un volumen comparable al de mercados importantes de países industrializados europeos como Inglaterra y Francia, habiendo superado incluso el mercado italiano en 1994. Este volumen abrió nuevas perspectivas para llegar a economías de escala en la producción y para el ingreso de nuevos productores. El cuadro muestra además el extraordinario dinamismo de los mercados de países de industrialización reciente, en comparación con la relativa estabilidad o declinación de los de países industrializados. Este es un punto que cabe destacar para entender las razones que han motivado un nuevo ciclo de inversiones en el sector. ${ }^{1}$

\footnotetext{
${ }^{1}$ La ANFAVEA no publica estadísticas relativas a los demás países del Mercosur. Se estima, sin embargo, que el consumo anual de vehículos es de unas 25000 unidades en Uruguay y de 15000 unidades en Paraguay (Folha de São Paulo, 1995).
} 
CUADRO 1

Algunos países desarrollados y en desarrollo: Licencias de vehículos nuevos, 1990-1995

\begin{tabular}{|c|c|c|c|c|c|}
\hline Año/ países & Brasil/Argentina & República de Corea & Francia & Reino Unido & Italia \\
\hline $\begin{array}{l}1990 \\
1991 \\
1992 \\
1993 \\
1994 \\
1995\end{array}$ & 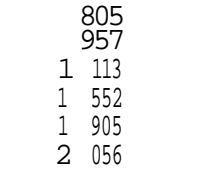 & $\begin{array}{rr} & 957 \\
1 & 106 \\
1 & 270 \\
1 & 438 \\
1 & 560 \\
1 & 563\end{array}$ & $\begin{array}{ll}2 & 756 \\
2 & 424 \\
2 & 466 \\
2 & 077 \\
2 & 299 \\
2 & 288\end{array}$ & $\begin{array}{ll}2 & 231 \\
1 & 801 \\
1 & 795 \\
1 & 975 \\
2 & 139 \\
2 & 195\end{array}$ & $\begin{array}{ll}2 & 483 \\
2 & 409 \\
2 & 573 \\
1 & 828 \\
1 & 789 \\
1 & 868\end{array}$ \\
\hline
\end{tabular}

Fuente: Asociación Nacional de Fabricantes de Vehículos Automotores (ANFAVEA).

¿Qué factores determinaron el repunte del crecimiento y el comienzo de la materialización del potencial del mercado brasileño? Los principales fueron la adopción sistemática de políticas de estímulo y sustentación de la demanda y, a partir de 1994, los efectos de la estabilización. Entre 1992 y 1994, la expansión de consumo obedeció sobre todo a la reducción de precios debida primordialmente a la caída de la tributación de los automóviles; ésta fue especialmente significativa en el caso de los automóviles "populares", ya que apenas decretada la medida comenzaron a llegar al mercado a unos 7500 dólares, precio atrayente para las condiciones del mercado de entonces. Entre 1993 y 1994, según datos de ANFAVEA, las ventas de vehículos populares experimentaron un crecimiento de $110 \%$, transformándose desde entonces en el puntal de la expansión de la demanda. La reapertura de los consorcios contribuyó también a dicha expansión, pero el papel del crédito directo al consumidor se vio menguado por las altas tasas de interés vigentes entonces y la inseguridad que suponía la inflación para el endeudamiento familiar. Ya en la fase del Plan Real, los factores que pesaban sobre la continuidad del crecimiento del mercado interno invirtieron su orden de importancia. La estabilización hizo más eficaces los mecanismos existentes de financiamiento directo al consumidor, ampliando sustancialmente el acceso de nuevos consumidores. Así, el consumo de automóviles (y de otros bienes durables) y el endeudamiento familiar crecieron juntos y se convirtieron en la marca característica de esta fase.

La tributación de los automóviles perdió su impacto después del Plan Real, dado que los precios de los vehículos automotores volvieron a subir neutralizando la reducción de alícuotas concedida por el gobierno desde 1992. Lo más notable fue el alza de precios de los vehículos "populares". Algunos de ellos subieron entre 53 y $65 \%$ entre agosto de 1994 y octubre de 1996, mientras el índice de precios al por mayor (IPM) subía $27 \% .^{2}$ Todo parece indicar que las ensambladuras se valieron de la protección adicional temporal concedida por el Régimen Automotor ${ }^{3}$ para recuperar la porción de los márgenes unitarios de la que habían desistido en la etapa de las cámaras sectoriales. Las ensambladuras alegan en su defensa que los aumentos son el resultado de la incorporación de costos adicionales incurridos en el cambio de modelos que se verificó en el período.

Pese al reciente aumento de precios, las ventas de vehículos "populares" siguieron expandiéndose y liderando el crecimiento del mercado interno. La participación de tales vehículos en el mercado interno ha sido continua desde su lanzamiento y alcanzó tal magnitud que configura una especialización del mercado en automóviles de este tipo. Dicha participación llegó a $56.3 \%$ en 1996 y la expectativa del mercado es que llegue a $60 \%$ en 1997. Tras este incremento hay aspectos estructurales importantes que repercuten sobre la inversión y la producción. El cambio descrito volvió a acercar el mercado automovilístico brasileño al patrón adecuado para el nivel de ingreso del país: un mercado de volumen, de automóviles de pequeño tamaño para la clase media. Las ensambladuras reaccionaron positivamente y alimentaron esta tendencia, in-

\footnotetext{
${ }^{2}$ La cuestión del alza de los precios internos de los vehículos automotores terminó por constituirse en un punto de conflicto en las relaciones de las ensambladuras con el gobierno y los revendedores autorizados, después de las alzas anunciadas en enero y marzo de 1997.

${ }^{3}$ El término designa la política gubernamental para el sector automotor, la cual después de la drástica elevación de las alícuotas de importación de automóviles en febrero y marzo de 1995, establece en esencia la reducción del impuesto a la importación de vehículos, máquinas y equipos e insumos para las empresas inscritas en el Régimen Automotor y como contrapartida exige el cumplimiento de metas de exportación y de índices de nacionalización en la compra de bienes de capital, materias primas y piezas y partes.
} 
cluso con el lanzamiento de modelos "populares" actualizados en los últimos dos años. La especialización del mercado constituyó la fuerza decisiva para que se dieran las tasas de crecimiento ya mencionadas, además de inducir también una especialización de la producción que contribuyó a aumentar las escalas.

\section{III \\ Repunte de la inversión y modernización tecnológica}

El repunte del crecimiento del mercado interno, las perspectivas dinámicas de crecimiento futuro y la especialización de este mercado incentivaron el incremento de la tasa de inversión de las ensambladuras. Esta tendencia se vio reforzada después que el gobierno dio a entender, mediante la adopción del Régimen Automotor, que no estaba dispuesto a absorber déficit comerciales importantes y crecientes en este sector. El comportamiento de las inversiones declarado por ANFAVEA muestra dos momentos de alza del nivel de inversión entre 1985 y 1995 (cuadro 2).

Entre 1985 y 1989, la inversión media de las empresas asociadas a ANFAVEA fue de 730 millones de dólares anuales, lo que corresponde a una tasa media de $4.3 \%$ de su valor de facturación. Entre 1990 y 1994 (inclusive) la inversión media anual creció cerca de $40 \%$, llegando a 1000 millones de dólares, lo que equivale a una tasa media de $6 \%$. Como se verá más adelante, la apertura comercial precipitó los planes de modernización productiva y renovación de la gama de productos de las ensambladuras, a los que correspon-

CUADRO 2

Brasil: Facturación e inversión en la industria automovilística ensambladora, 1985-1995

(En millones de dólares)

\begin{tabular}{lccc}
\hline Año & Facturación & Inversión & $\begin{array}{c}(\mathrm{A} / \mathrm{B}) \\
\frac{\circ}{0}\end{array}$ \\
\hline 1985 & & & 4.1 \\
1986 & 16282 & 674 & 4.7 \\
1987 & 15608 & 728 & 4.6 \\
1988 & 16777 & 773 & 3.9 \\
1989 & 18941 & 735 & 4.2 \\
1990 & 17562 & 918 & 7.0 \\
1991 & 13096 & 981 & 7.3 \\
1992 & 13462 & 983 & 5.9 \\
1993 & 16718 & 939 & 4.8 \\
1994 & 19369 & 1230 & 5.2 \\
1995 & 23542 & 1694 & 6.9 \\
\hline
\end{tabular}

Fuente: Asociación Nacional de Fabricantes de Vehículos Automotores (ANFAVEA). dio el incremento de la tasa de inversión. A partir de 1994, el aumento de la inversión sugiere un nuevo cambio de nivel. La inversión llegó casi a 1700 millones de dólares en 1995 (tasa de 6.9\%), y los indicadores apuntan a que continuará creciendo hasta el final de los años noventa.

La expansión de la capacidad en el primer lustro de esta década derivó sobre todo de la inversión que se destinó a modernizar las plantas existentes. El lanzamiento de nuevos modelos basados en proyectos que simplifican la producción, la adopción de nuevas tecnologías de fabricación y la difusión de conceptos y técnicas de organización inspirados en la experiencia japonesa elevaron la productividad de las fábricas, ampliando su capacidad. Sin embargo, en el segundo lustro de los años noventa el aumento de la capacidad se dará también en función de las nuevas plantas en construcción, no sólo de las ensambladuras que ya están en el mercado sino también de las que ingresen posteriormente. Por lo tanto, dichas inversiones deberán sumarse a las inversiones en modernización. Las inversiones anunciadas y comprometidas en el ámbito del Régimen Automotor por los competidores actuales y potenciales para la instalación de nuevas plantas ascienden a 3600 millones de dólares, suma que se distribuirá desde 1996 hasta el año 2000 (cuadro 3). Este conjunto de nuevas fábricas aumentará la capacidad de producción de la industria automovilística brasileña en alrededor de 600000 vehículos. Si sólo se considera esta capacidad adicional sumada al nivel actual de producción, se llega a un volumen de 2.4 millones de unidades/año, que se alcanzaría el año $2000 .^{4}$

\footnotetext{
${ }^{4}$ En la práctica, este total debe superarse, dado que la capacidad y la producción de algunas de las actuales líneas de producción tienen que ampliarse en los próximos años. Este es el caso, por ejemplo, de la fabricación de los modelos Fiesta, Palio y Novo Gol.
} 
CUADRO 3

Brasil: Inversiones confirmadas en nuevas plantas de vehículos automotores, 1996-2000

\begin{tabular}{llllll}
\hline Empresa & $\begin{array}{l}\text { Inversión } \\
\text { (millones } \\
\text { de dólares) }\end{array}$ & Ubicación & $\begin{array}{l}\text { Capacidad } \\
\text { (unidades) }\end{array}$ & $\begin{array}{l}\text { Modelo } \\
\text { la producción }\end{array}$ \\
\hline Fiat & $150-200$ & Belo Horizonte (MG) & 100000 & Vehículos comerciales livianos \\
GM & 600 & Gravataí (RS) & 120000 & Vehículos "populares" por definir \\
Mercedes & 400 & Juiz de Fora (MG) & 70000 & Classe A & 1999 \\
Renault & 750 & S.J. dos Pinhais (PR) & 100000 & Mégane \\
Toyota & 150 & Indaiatuba (SP) & 15000 & Corolla \\
Honda & 100 & Sumaré (SP) & 15000 & Civic \\
Chrysler & 315 & Campo Largo (PR) & 40000 & Dakota \\
BMW & 150 & S.J. dos Pinhais (PR) & 60000 & Defender \\
Audi/Volks & 500 & Camaçari (BA) & 60000 & Audi A3 y VW Vento \\
Asia & 500 & & Topic y Towner & 1999 \\
\hline
\end{tabular}

Fuente: Archivos de Folha de Säo Paulo y Gazeta Mercantil.

CUADRO 4

Brasil: Escalas de producción en las ensambladoras por principales familias de automóviles, 1990-1995

(En unidades)

\begin{tabular}{|c|c|c|c|c|c|c|}
\hline EmpresaX^año & 1990 & 1991 & 1992 & 1993 & 1994 & 1995 \\
\hline Familia Uno (Fiat) & 131372 & 136640 & 134131 & 183999 & 251952 & 266501 \\
\hline Familia Escort (Ford) & 79928 & 86138 & 63391 & 138152 & 157080 & 152215 \\
\hline Familias Chevette/ & & & & & & \\
\hline Corsa (GM) & 26632 & 26841 & 41034 & 32773 & 68304 & 156573 \\
\hline Familia Gol (vw) & 138029 & 147167 & 186254 & 205304 & 225911 & 304457 \\
\hline
\end{tabular}

Fuente: Asociación Nacional de Fabricantes de Vehículos Automotores (ANFAVEA).

A la par con el crecimiento de las ventas la producción brasileña de vehículos automotores dio un salto significativo a partir de 1993, sobrepasando las 1.8 millones de unidades en 1996 (es decir, creció casi $100 \%$ en relación con el nivel de 1990). El incremento de la producción automovilística brasileña y su especialización en vehículos de tamaño pequeño permitió otro cambio estructural significativo en relación con el cuadro que predominaba hasta comienzos de los años noventa. Por primera vez, todas las ensambladoras de automóviles alcanzaron escalas óptimas de producción para sus modelos más vendidos. Según la evaluación de O'Brien y Karmakolias (1994, p. 21), las escalas óptimas en el montaje de vehículos automotores varían entre 100000 y 200000 unidades/año, según el tipo de vehículo. En las ensambladoras brasileñas las escalas de producción de los automóviles pequeños crecieron sustancialmente a partir de 1993, año en que se inició la producción y venta de los vehículos populares. En 1995 las escalas ya habían superado las 150000 unidades/año para las principales familias de modelos: Gol, Uno, Corsa y Escort (cuadro 4).
Sin embargo, la continuidad de los aumentos de escala puede verse amenazada a futuro por los cambios estructurales del mercado automovilístico que provocará la entrada de nuevos competidores. En un panorama general de la estructura actual del mercado automovilístico (gráfico 2), dos puntos llaman la atención. Primero, la irrelevancia de la participación de otras marcas aparte de aquellas que han concentrado la producción de automóviles en Brasil por más de una década: Volkswagen, General Motors, Ford y Fiat. Y segundo, el notable crecimiento de la participación de la Fiat en el mercado a partir de 1990, derivado de su apuesta por el vehículo "popular", y en detrimento sobre todo de la Ford, que creyó menos en el mercado brasileño.

De hecho, la estrategia de crecimiento y de búsqueda de liderazgo del mercado aplicada por la Fiat puede interpretarse como el primer factor importante en desestabilizar el oligopolio que caracterizó al sector por tanto tiempo. Un cambio significativo que viene con las nuevas plantas es la entrada de siete competidores nuevos al mercado automovilístico brasile- 
GRÁFICO 2

Brasil: Participación de las empresas asociadas a ANFAVEA en el mercado interno de automóviles (producción nacional), 1985-1996
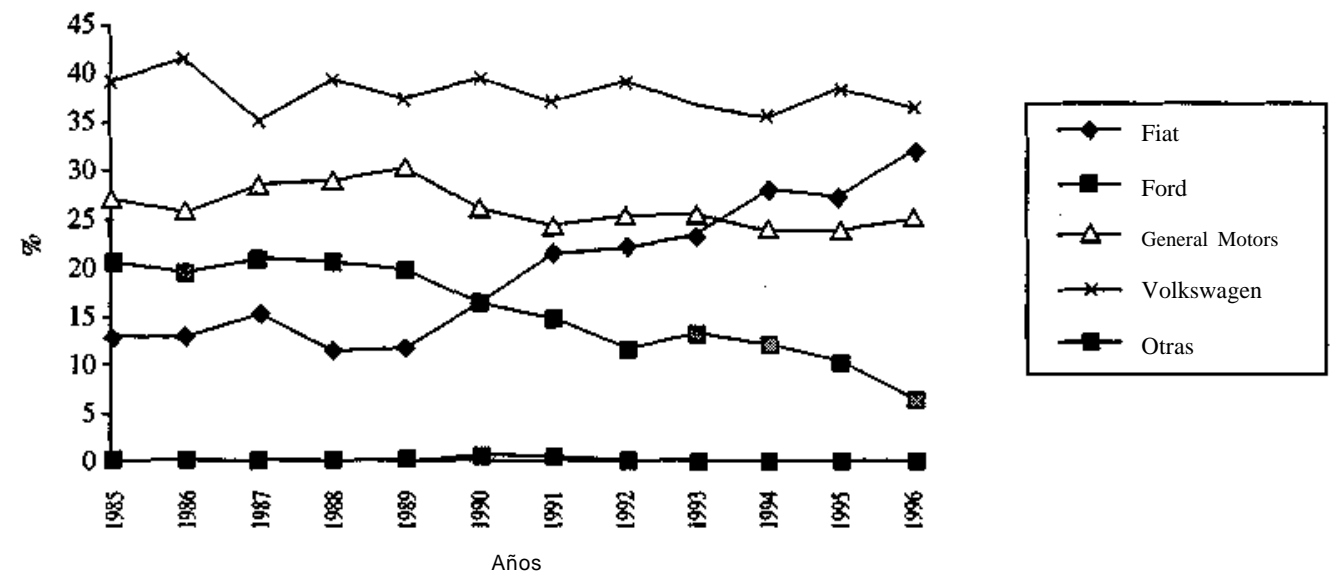

Fuente: ANFAVEA.

ño, de los cuales dos son japoneses (Toyota y Honda), dos alemanes (BMW y Audi), uno estadounidense (Chrysler), uno francés (Renault) y uno coreano (Asia). ${ }^{5}$ A lo anterior cabe agregar la producción de automóviles de la Mercedes Benz que, pese a estar instalada hace mucho tiempo en el país, sólo fabrica camiones y omnibuses a nivel local. Por lo tanto, se va a modificar la posición marginal de otras marcas respecto a las cuatro grandes de hoy. La reducción del nivel de concentración en la industria automovilística puede acarrear como beneficio la oferta de productos más baratos y/o de mayor calidad. El ingreso de nuevos competidores contribuye a reducir el poder de mercado de las empresas instaladas y la competencia resultante puede generar beneficios para los consumidores. Empero, los nuevos competidores deberán aumentar una capacidad de producción que ya es sustancial. La fragmentación excesiva del mercado puede llegar a amenazar los beneficios de escala proporcionados por el crecimiento reciente, reeditando el mayor obstáculo de los países en desarrollo para alcanzar escalas óptimas de producción. Seguir haciendo hincapié en la especialización es importante para minimizar ese riesgo.

Otro cambio importante en la industria automovilística brasileña de los años noventa es el mayor

\footnotetext{
5 Además de la entrada de esos nuevos competidores con compromisos de inversión asumidos, ha habido especulaciones respecto a la entrada de la Peugeot (francesa), la Mitsubishi (japonesa), la Daewoo y la Hyundai (coreanas).
}

dinamismo tecnológico. En esta década, las inversiones realizadas por la industria ensambladura se orientaron a la renovación casi completa de la gama de productos, así como a la adopción de nuevos procesos y nuevas formas de gestión inspirados en la experiencia japonesa y a la reestructuración de las relaciones con los proveedores de piezas y partes y otros insumos.

Una evaluación somera de la evolución de los lanzamientos de modelos básicos ${ }^{6}$ por las ensambladoras de automóviles desde 1990, revela un pronunciado aumento de los nuevos lanzamientos en la década, sobre todo después de 1992 (gráfico 3): ya se han lanzado 14 modelos nuevos, contra sólo siete en los años ochenta. Un aspecto que cabe destacar en esta renovación de productos es el cierre de la brecha entre la edad de los productos fabricados y comercializados en Brasil y la de los lanzados más recientemente por las ensambladuras en los países más industrializados. A partir de 1992, casi todos los lanzamientos consistieron en automóviles "mundiales" actualizados, o sea, modelos que son producidos concomitantemente para los mercados más exigentes. Modelos como el Corsa, el Palio y el Fiesta son ejemplos de esta tendencia que deberá acentuarse.

Junto con la renovación de sus productos las ensambladoras brasileñas promovieron una moderniza-

\footnotetext{
${ }^{6}$ Se considera sólo el lanzamiento de modelos nuevos con plataforma propia, omitiendo el lanzamiento de modelos nuevos de una misma plataforma/familia.
} 


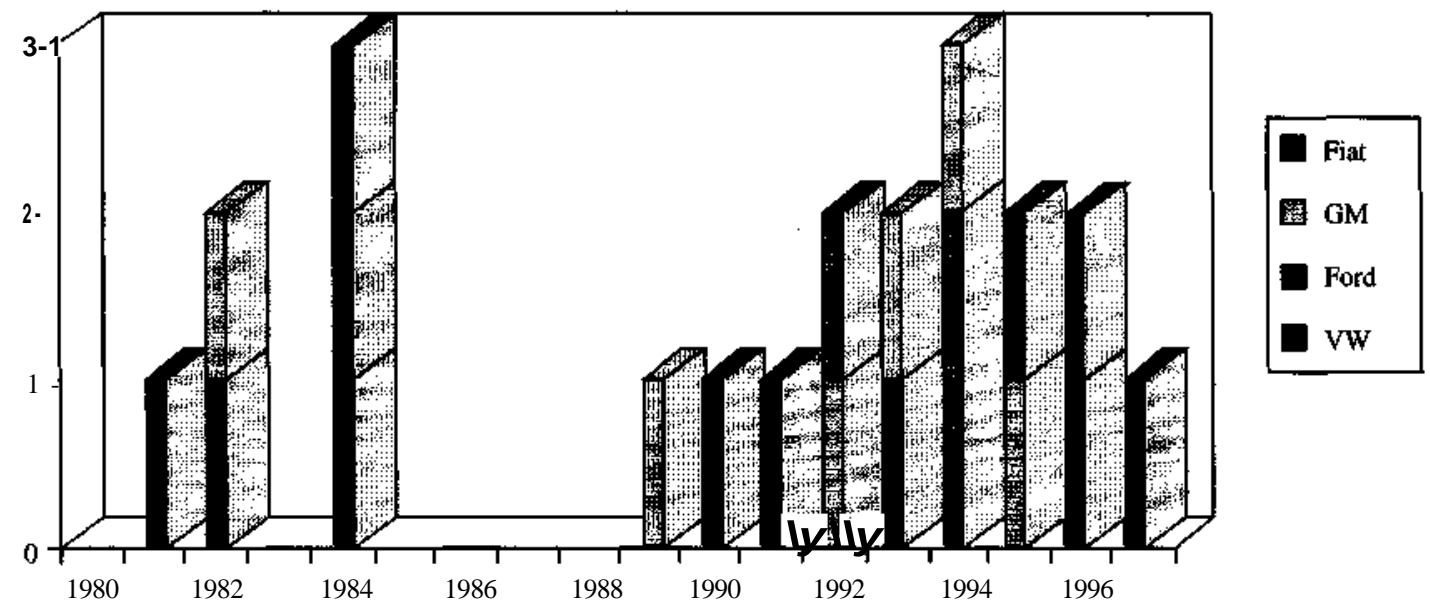

Fuente: Archivos de Folha de São Paulo y Gazeta Mercantil.

ción sustancial de sus métodos productivos y prácticas de organización y gestión, y modificaron sus relaciones con los proveedores. La renovación de modelos fue seguida muy pronto por la incorporación de la automatización programable en la industria automovilística brasileña. Especialmente en las líneas de producción de alto volumen de los modelos nuevos de automóviles pequeños se incorporó un número importante de robots. ${ }^{7}$ Nuestra estimación, basada en fuentes del mercado, es que la existencia de robots en las fábricas de automóviles brasileñas se aproxima hoy a las 500 unidades. Aunque este número es reducido en relación con el tamaño de la industria, ${ }^{8}$ la adopción selectiva de la automatización programable se ha acelerado. La difusión de tecnologías de automatización programable contribuye a la consistencia de la calidad de los productos en este sector.

Otro punto que cabe destacar es el cambio de los conceptos y técnicas de gestión. Las informaciones y publicaciones disponibles sugieren que más intensa que la difusión de la automatización programable ha sido la difusión de nuevas técnicas de organización de la producción y del trabajo en las ensambladuras brasile-

${ }^{7}$ Este es el caso de las líneas: Corsa, Novo Gol y Fiesta.

${ }^{8}$ La difusión de los robots, más que la de otras tecnologías de automatización programable, está determinada en gran medida por el costo de la mano de obra. Esto explica por qué la tasa de difusión en Brasil todavía es limitada. En la República de Corea se llegó a tener 500 robots en 1985. ñas. Nos referimos a nuevos principios de administración y técnicas de organización de inspiración japonesa como las de producción justo a tiempo (impulsada por la demanda), de calidad total, y el principio y las técnicas del Kaizen (mejora continua). No hay investigaciones exhaustivas recientes sobre la difusión de esas innovaciones en el conjunto de la industria automovilística. No obstante, los estudios de caso existentes señalan que, con ritmos y enfoques diferentes, la mayor parte de las ensambladuras avanzó considerablemente en la incorporación de dichas técnicas. ${ }^{9}$

Las relaciones establecidas por las ensambladuras con sus proveedores de piezas y partes y otros insumos son otro aspecto importante. En general, las ensambladoras están modificando considerablemente sus relaciones con los proveedores de piezas y partes. La base del cambio ha sido una exigencia apremiante de mejorar la calidad y reducir los costos. El aumento de las exigencias en materia de calidad y plazos de entrega y de la presión sobre los márgenes de ganancia de los productores de piezas y partes no es un fenómeno exclusivo de Brasil. Éste se intensificó con las prácticas conocidas como global sourcing. El hecho de que este cambio se identifique como el "efecto López" en la jerga del sector, ilustra no sólo la importancia del ex

\footnotetext{
${ }^{9}$ Respecto al cambio organizacional reciente en la General Motors y en la Volkswagen de Brasil, véase Fleury y Salerno (1995). Para un resumen de los cambios más importantes en la Fiat de Brasil, véase Quadros Carvalho y Bernardes (1997).
} 
ejecutivo de la General Motors y de la Volkswagen como deflagrador de este proceso, sino también el carácter global del cambio. Tal vez el ejemplo más innovador en términos de relaciones con los proveedores haya sido la adopción por la Volkswagen del llamado "consorcio modular" para la producción de camiones en la fábrica de Resende.

La modernización de productos y procesos se reflejó en el aumento de productividad de la industria ensambladura. La prensa ha explotado innumerables veces el hecho de que la producción física de vehículos por empleado para el conjunto de la industria se duplicó con creces entre 1990 y 1995, pasando de 7.8 a 15.6 vehículos. Sin embargo, este cálculo nos parece sesgado, habida cuenta de los profundos cambios estructurales que analizamos a lo largó del presente estudio. En él no se consideran dos cambios fundamentales en la estructura de la producción. Primero, en la producción de automóviles subió la participación de los vehículos pequeños y, sobre todo, de los vehículos "populares" (vehículos de menor valor), de modo que a partir de 1993 la producción física no puede compararse con la del período 1989-1992, a no ser que se aplique alguna forma de cálculo de unidades equivalentes. Segundo, y más importante aún, el movimiento de "externalización de la producción", del que la política de "crecimiento hacia afuera" de la Fiat resulta emblemática, implicó la transferencia a los proveedores de las ensambladuras de un conjunto de actividades manufactureras que antes estaban a cargo de éstas. Así, la estructura de la producción a mediados de los años noventa ya parecía diferir de la estructura con la que se había iniciado la década. Por lo tanto, en ese caso es válida la crítica de que la "tercerización" sesga los cálculos de productividad física.

El índice de facturación por empleado, descontado el efecto de las importaciones, presenta un crecimiento mucho más modesto, de $36 \%$, comparando los años 1994 y 1989. Este indicador también es problemático porque además de mantener el sesgo de la tercerización introduce algunas distorsiones adicionales. En vista de ello, realizamos un ejercicio para neutralizar parcialmente el efecto tercerización del índice de facturación, producción nacional por empleado, mediante la sustracción del consumo de piezas y partes importadas o producidas por las empresas asociadas a Sindipecas. Adoptando este indicador el crecimiento de la prodúctividad para el trienio 1993-1995 fue de 35\% comparado con el trienio anterior. Se trata de un crecimiento significativo, pero muy inferior al de la relación producción de vehículos/empleo.

En suma, este análisis nos lleva a dos conclusiones principales. Primero, dado que todos los indicadores apuntan en la misma dirección y considerando también otros indicadores de eficiencia mencionados en este estudio, hay pruebas de que hubo aumentos significativos de la productividad de las emsambladoras en el período examinado. No obstante, éstos son inferiores al aumento de productividad que ha sido divulgado al público. Segundo, las informaciones disponibles en las bases sectoriales no permiten sacar conclusiones precisas sobre la magnitud de dichos aumentos. Esto apunta a la necesidad de efectuar investigaciones adicionales que persigan obtener de las empresas las informaciones necesarias para superar los sesgos ya señalados.

\section{IV}

\section{La nueva inserción externa del sector automotor}

El tercer conjunto de cambios que analizamos en este artículo está relacionado con la inserción internacional de la industria automovilística brasileña. Tal inserción fue modificada sustancialmente en un primer momento por la apertura comercial, y luego por la adopción del Régimen Automotor. Brasil dejó de ser uno de los mercados más cerrados del mundo a la importación de vehículos automotores, y se redujo también el índice medio de nacionalización de productos terminados (superior a $95 \%$ antes de la apertura). Pero pronto se observó que la capacidad de la economía brasileña de absorber déficit considerables en el comercio de vehículos y piezas y partes era limitada. Estos límites indujeron al gobierno a adoptar el Régimen Automotor. Las señales que éste enviaba contribuyeron a su vez a inducir inversiones en la producción local especializada, abriendo tal vez nuevas perspectivas para exportaciones futuras. En este cuadro de reacomodo de los volúmenes y flujos comerciales se ha destacado el crecimiento del comercio con los países del Mercosur, en especial con Argentina, lo que refleja la consolidación del mercado regional. 
CUADRO 5

Brasil: Balanza comercial de vehículos automotores, piezas y partes, 1989-1996

(En millones de dólares fob corrientes)

\begin{tabular}{lcccccccc}
\hline Productos & 1989 & 1990 & 1991 & 1992 & 1993 & 1994 & 1995 & $1996^{\mathrm{a}}$ \\
\hline $\begin{array}{l}\text { Omnibuses } \\
\text { Automóviles y vehículos } \\
\text { comerciales livianos }\end{array}$ & 127 & 57 & 118 & 269 & 329 & 206 & $(14)$ & $(7)$ \\
$\begin{array}{l}\text { Camiones } \\
\begin{array}{l}\text { Total de vehículos } \\
\text { automotores }\end{array}\end{array}$ & 681 & 406 & 226 & 494 & $(78)$ & $(935)$ & $(2584)$ & $(821)$ \\
$\begin{array}{l}\text { Piezas y partes } \\
\text { Total del sector }\end{array}$ & 1593 & 437 & 331 & 538 & 301 & 302 & $(190)$ & 112 \\
& 1308 & 1185 & 1260 & 1536 & 1511 & 1282 & $(2788)$ & $(716)$ \\
\end{tabular}

Fuente: Elaboración propia a partir del banco de datos de la Secex.

a Para el año 1996: exportaciones de enero/diciembre e importaciones de enero/octubre x 1.2.

La apertura comercial propició, por primera vez en la historia de la industria automovilística brasileña, una participación destacada de los vehículos importados en las ventas internas. El aumento de dicha participación desde los niveles muy bajos en que se encontraba hasta comienzos de los años noventa tiene un carácter estructural y no transitorio. La caída de esa participación de 21.3 a $12.9 \%$ entre 1995 y 1996 fue el resultado de las medidas de contención vinculadas al Régimen Automotor, y no debe interpretarse como un retorno a la condición anterior. Igual importancia ha tenido para la estructura de la industria el crecimiento de las importaciones de piezas y partes. Entre 1991 y 1996, su valor creció a una tasa media anual de $30.2 \%$, y su participación en el consumo brasileño total de piezas y partes se disparó de 5.6\% en 1989 a casi $21 \%$ en 1996.

El recurso a las importaciones de piezas y partes fue una de las estrategias adoptadas por las ensambladoras para hacer posible a corto plazo la renovación de la gama de productos y la mejora de la calidad de los vehículos. ${ }^{10}$

Entre 1990 y 1995 el arancel específico a la importación de vehículos automotores sufrió diversas alteraciones. ¿Cuáles fueron esas alteraciones y cómo reaccionaron las ensambladuras a dichos cambios? Entre mayo de 1990 y octubre de 1992, el arancel se redujo progresivamente de $85 \%$ a $40 \%$. Con la recuperación del mercado interno en 1993, la eliminación paulatina de los aranceles mostró su primer reflejo significativo. Ese año las importaciones de vehículos automotores crecieron en $150 \%$, muy por encima del

\footnotetext{
${ }^{10}$ Véase una evaluación más detallada de los efectos de la apertura en la industria brasileña de piezas y partes en Posthuma (1995).
}

crecimiento de $43 \%$ de la producción. La reducción arancelaria prosiguió hasta septiembre de 1994, momento que coincidió con la valorización cambiaria vinculada a la implantación del Plan Real, lo que estimuló notoriamente las importaciones. Una vez constatado el creciente desequilibrio externo generado en el sector automotor, el arancel de importación volvió a subir en febrero de 1995 y en forma más abrupta el mes siguiente, cuando volvió al nivel de $70 \%$, dando inicio a las negociaciones que culminarían con la implantación del Régimen Automotor. Las importaciones siguieron creciendo más que la producción nacional en el bienio 1994-1995 y menguaron en 1996 debido a las medidas adoptadas el año anterior. Las cifras pertinentes de la balanza comercial (cuadro 5) mostraron un rápido deterioro a partir de 1994, que se acentuó en 1995 y se revirtió en parte en 1996.

Estos vaivenes no podían dejar de repercutir sobre los planes de inversión de las empresas. Frente a un mercado en expansión y en apertura creciente, éstas buscaban el punto de equilibrio entre la producción interna y el abastecimiento externo. En un primer momento, hubo un repunte de la inversión en la ampliación de la producción y el lanzamiento de nuevos modelos para competir con los productos importados. Más adelante, las propias ensambladuras instaladas en el país se transformaron en las principales importadoras, deshancando a los importadores independientes y, frente a un panorama que estimulaba notoriamente las compras externas, pasaron a postergar sus planes más osados de expansión de la producción interna. Por último, tranquilizadas por las señales que enviaba el gobierno de que no asistiría impasible a un deterioro de las cuentas externas del sector automotor, las empresas reanudaron los programas de inversión. 
Dados los cambios estructurales ya comentados que vienen ocurriendo en la industria, cabe preguntarse hasta qué punto es razonable esperar un aumento de la competitividad que traiga efectos positivos para la balanza comercial y en qué plazo. A nuestro juicio, no se pueden esperar cambios significativos a corto plazo de la posición competitiva de la industria. Habrá que contener el déficit de la balanza comercial de este sector mediante las restricciones a las importaciones y los estímulos a las exportaciones previstos por el Régimen Automotor. Sin embargo, en el mediano plazo la especialización creciente y las inversiones en curso tenderán a favorecer las exportaciones. Frente a un mercado local y regional en expansión y bajo la vigilancia gubernamental del comportamiento de las cuentas externas, la estrategia de la mayoría de las empresas parece contemplar el aumento de la producción y de las exportaciones necesarias para compensar las importaciones que deberán complementar sus líneas de productos. Esto no significa que Brasil va a transformarse en una base exportadora importante para el mercado mundial - la motivación primordial de las inversiones sigue siendo la producción para el mercado interno y regional - pero permite prever iniciativas más sólidas de participación en el mercado externo a partir de las nuevas plantas y la modernización de las antiguas. Esta perspectiva es consistente con los resultados de una reciente investigación (Reis, Branco y Bielchowsky, 1997) relativa al conjunto de la industria brasileña, que muestra que el aumento del coeficiente de exportación depende, sobre todo, de la inversión motivada por un mercado interno en rápido crecimiento.

Por último, otro aspecto importante en la nueva inserción externa que se perfila para el sector automovilístico brasileño es el papel del Mercosur. La participación del Mercosur en las exportaciones de vehículos creció considerablemente a partir de 1991, y entre 1992 y 1996 se viene manteniendo en un nivel cercano a 50\%. Entre 1995 y 1996, las importaciones provenientes del Mercosur (es decir, Argentina) también registraron un aumento espectacular de 15 a 49\%. Estos datos reflejan el proceso de unificación del mercado regional resultante de la distribución entre Brasil y Argentina de las inversiones que realizan las ensambladoras. Estas empresas buscan obtener cierto equilibrio en el comercio intrarregional a la vez que consiguen aumentar la especialización y, por consiguiente, la eficiencia de sus plantas. La tendencia a ampliar el intercambio de productos terminados, piezas y componentes entre los países de la región se ha consolidado.

\section{V}

\section{El papel del Régimen Automotor}

La interrogante que pareció plantearse nítidamente a las empresas en el segundo semestre de 1994, tras adelantarse el cronograma de reducción arancelaria previsto para el Mercosur, fue la de definir la mejor combinación entre producción interna e importación a fin de ocupar ese mercado en expansión. Las vacilaciones observadas entonces, como los anuncios de planes de expansión seguidos de retrocesos y estudios de alternativas —el ejemplo de la tercera fábrica de la GM es ilustrativo - sugieren que las empresas todavía buscaban una respuesta adecuada a esa pregunta.

Cabe subrayar dos hechos importantes al respecto. Primero, las ventas crecieron más que la producción durante el período 1992-1995 y la participación de los vehículos importados aumentó considerablemente, presionando excesivamente la balanza comercial del sector. Segundo, las empresas instaladas en el país reaccionaron rápidamente a la entrada de los importado- res independientes y pasaron a ser ya en 1993 las principales vendedoras de vehículos importados. El primer hecho plantea la posibilidad de que la composición de "equilibrio" entre producción interna y exportaciones que buscaban las empresas pudiera situarse en un punto correspondiente a un fuerte déficit de la balanza comercial del sector. El segundo muestra los límites de la apertura comercial como mecanismo capaz de afectar las posiciones dominantes de las empresas líderes, al menos en el corto plazo.

El Régimen Automotor desempeñó un papel decisivo como catalizador de las decisiones de inversión que tomaron las empresas a contar de 1995. Esto obedeció no tanto a los beneficios que estipulaba el Régimen —que duran sólo hasta 1999 - sino porque el gobierno dio a entender que no admitiría un desequilibrio comercial elevado y permanente, lo que dada la gravitación del sector automotor termina por repercu- 
tir sobre la balanza comercial brasileña. Así, se hizo creer a las empresas que la relación de "equilibrio" producción interna/importaciones debería ser más cercana a aquella que también permite un mayor equilibrio del comercio sectorial. Ese efecto sobre la inversión fue el resultado más importante del Régimen Automotor. En 1995 la inversión de las empresas afiliadas a ANFAVEA se disparó a una cifra cercana a los 1700 millones de dólares, comparada con una media de aproximadamente 1000 millones de dólares en el quinquenio anterior. Además, ya están confirmadas solicitudes de inversión que sobrepasan los $3600 \mathrm{mi}-$ llones de dólares, provenientes en su mayor parte de empresas recién llegadas, sólo para la instalación de nuevas plantas, las que van a aumentar la capacidad en unos 600 mil vehículos en los próximos tres años. Todos esos planes, cuya ejecución en realidad ya se inició, contemplan la construcción de plantas modernas y la fabricación de modelos actualizados.

Como resultado de esas inversiones cabe esperar una modernización más acelerada del sector automotor. La renovación de productos habida en el último quinquenio redujo el hiato entre la edad de los productos fabricados y comercializados en Brasil y la de los lanzamientos más recientes de las ensambladuras en el exterior. Esta tendencia va a acentuarse. Lo mismo será válido para las innovaciones en los procesos y las relaciones con los proveedores. La nueva fábrica de camiones de la Volkswagen en Resende (Rio de Janeiro) promete hacer del llamado "consorcio modular" un marco significativo en el sector automovilístico. A su vez, la General Motors ya anunció para la nueva planta de Gravataí (Rio Grande do Sul) la intención de implantar un sistema con características semejantes, denominado condominio industrial. Todo esto va a tornar más nítidos los aumentos de productividad del sector, que han sido difíciles de estimar y muy probablemente menos acentuados de lo que se ha dicho.

Así, los adelantos propiciados por las nuevas inversiones en procesos y productos deberían mejorar la competitividad del sector automotor, lo que a su vez habrá de reflejarse positivamente en la balanza comercial. Las plantas, además de más modernas son cada vez más especializadas, lo que permite un mejor aprovechamiento de las economías de escala. El volumen de producción que las ensambladuras principales ya logran obtener, ampliado por las nuevas inversiones, refuerza la competividad externa. Dentro de esa perspectiva, el Régimen Automotor contribuye a mejorar las cuentas externas, frenando las importaciones y ampliando las exportaciones mediante incentivos en el corto plazo y favoreciendo principalmente las exportaciones en función de la mayor competitividad en el mediano plazo.

Cabe subrayar además el papel del Régimen Automotor en el desarrollo industrial y tecnológico más amplio. Se sabe que la industria automovilística tiene importantes efectos de eslabonamiento que multiplican los beneficios derivados de las decisiones de inversión de las ensambladuras y sus socios más próximos del sector de piezas y partes. A título ilustrativo, podemos citar la inversión recién aprobada de 1000 millones de dólares anunciada por Usiminas para suplir hasta 1999 el aumento previsto de la demanda de aceros nobles con la llegada de nuevas ensambladuras. Además, estas empresas definen un patrón complejo de organización de la producción y de desarrollo tecnológico que repercute sobre los demás segmentos de la trama industrial.

Sin embargo, el Régimen Automotor mostró aspectos controvertidos. El señalado con mayor frecuencia es el trato desigual que da a las ensambladuras en relación con los productores de piezas y partes. De hecho, los niveles de protección establecidos para la industria automovilística son excepcionalmente elevados y han permitido que las ensambladuras abusen de los aumentos de precios y aumenten su rentabilidad, ya bastante subida antes de la vigencia de dicho Régimen. Pero ese cuadro es transitorio, no sólo porque la protección es temporal sino porque la ampliación de la capacidad instalada prevista para los próximos años obligará a las empresas a reducir los márgenes para ampliar el mercado. En todo caso, queda la duda sobre el "calibre" adecuado de los niveles de protección efectivo de las ensambladuras. Se trata de determinar el punto correcto entre el $20 \%$ de arancel nominal, que reduciría gran parte de la inversión programada, y el $70 \%$ que permite márgenes de utilidad excesivos en perjuicio del consumidor. $\mathrm{O}$, en su defecto, encontrar medidas efectivas de defensa de la competencia que frenen los abusos por parte de las ensambladuras.

A su vez, la industria de piezas y partes se halla frente a un cuadro de márgenes de utilidad escasos y un mercado amenazado por las importaciones. Cabe observar que el proceso de reestructuración de este segmento se diferencia del analizado para las ensambladoras, pues guarda escasa relación con el Régimen Automotor. Los cambios en marcha en la industria de piezas y partes tienen una dimensión global que, sumada a la apertura económica brasileña, está promoviendo una rápida concentración y desnacionalización. 
Los factores que explican esta situación son: i) las exigencias crecientes de las ensambladoras en términos de precio, calidad y plazo de entrega; ii) la tendencia de esas mismas empresas a trabajar con un número reducido de proveedores, y iii) relacionado con lo anterior, el recurso al ensamblaje compartido, es decir, la preferencia de las ensambladoras por el suministro de sistemas o conjuntos de piezas ya montados y no de componentes individuales.

Pese a que las intenciones de las ensambladoras de ampliar las importaciones de componentes se han revelado más una amenaza que una realidad, sus exigencias han producido una fuerte presión competitiva sobre las empresas proveedoras. Aunque aún no estén claros el sentido y el alcance de los cambios que recién comienzan en la industria de piezas y partes, es posible observar que determinadas actividades tienden a suprimirse, que las sociedades tecnológicas y comerciales con empresas líderes mundiales adquieren un papel estratégico, y que la obtención de patrones de calidad y de productividad internacionales pasa a ser una prioridad. Se precisan estudios más acabados para establecer la naturaleza y consecuencias de la reestructuración del sector de piezas y partes.

Senáa prematuro pretender realizar ahora un balance del Régimen Automotor. No obstante, este artículo ha aclarado bastante los beneficios ya obtenidos: en el corto plazo, se evita un aumento del déficit en la balanza comercial y, en el mediano plazo, a partir de las inversiones inducidas se acelera la modernización de la industria automovilística y se refuerza también la competitividad externa. Se han señalado asimismo los problemas principales, sobre todo el trato desigual otorgado a las ensambladoras y a los fabricantes de piezas y partes y el encarecimiento de los automóviles, a lo menos en el corto plazo. Por ahora, es razonable concluir que un proceso de índole compleja y de consecuencias dolorosas como es el de la apertura comercial necesita recurrir a políticas pragmáticas que permitan una administración consecuente de esta fase de transición.

(Traducido del portugués)

\section{Bibliografía}

Fleury, A. y M. Salerno (1995): Hybridisation of industrial models in the Brazilian automobile industry. Anuales du Troisiéme Rencontre Internationale: Les nouveaux modeles industriéis, Paris, Gerpisa.

Folha de São Paulo (1995): Caderno especial São Paulo, 26 de enero.

O'Brien, P. e Y. Karmakolias (1994): Radical reform in the automobile industry: policies in emerging markets, $\mathbb{F C}$ discussion paper, $\mathrm{N}^{\circ} 21$, Washington, D.C. Banco Mundial.

Posthuma, A.C. (1995): Restructuring and changing market conditions in the Brazilian auto components industry, LC/R.1484, Santiago de Chile, Comisión Económica para América Latina y el Caribe (CEPAL).

Quadros Carvalho, R. y R. Bernardes (1997): Mudando com a economía: estrategias de ajuste de empresas líderes brasileiras, Campinas, estudio preparado para la División de Desarrollo Productivo y Empresarial de la CEPAL, mimeo.

Reís, J., F. Branco y R. Bielschowsky (1997): Investimentos na industria brasileira 1995/1999, Rio de Janeiro, Confederación Nacional de la Industria. 